\title{
CONSIDERACIONES DE DISEÑO
}

Manuel Thomé de Guezala, Ingeniero de Caminos*

Marc Quivrin, Ingénieur Chimiste**

\section{CONSIDERACIONES GENERALES}

El tanque de almacenamiento de GNL del Terminal de Palos de la Frontera es un depósito autoportante del tipo doble pared hormigón-acero, compuesto por un depósito exterior de hormigón doblemente postesado y un depósito interior de acero $9 \%$ Níquel, con techo suspendido de aluminio (Fig. 1).

El diseño del tanque responde a las exigencias de proyecto y a los condicionantes de su emplazamiento que se resumen a continuación.

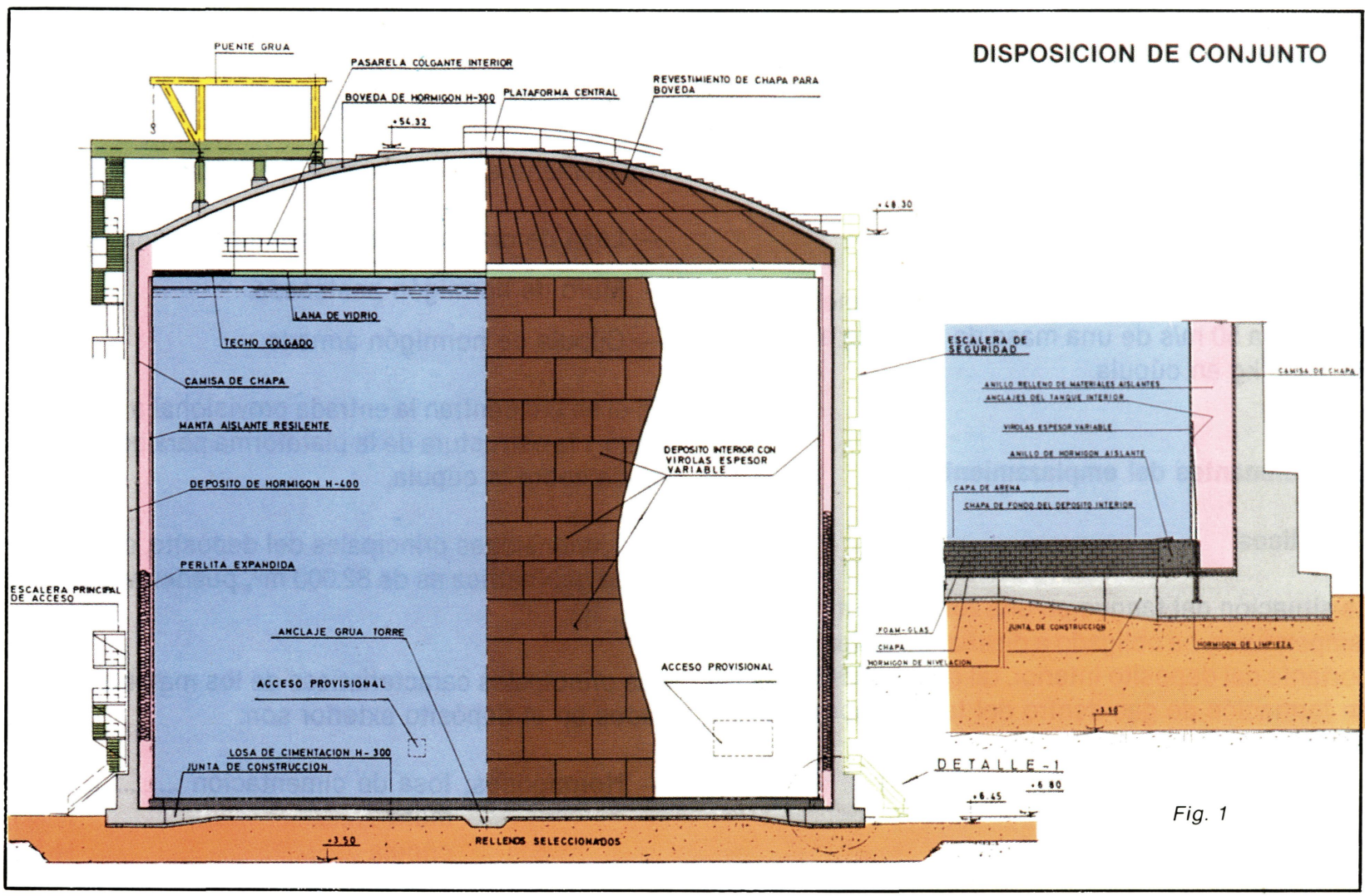

\section{Exigencias de proyecto:}

Capacidad neta $60.000 \mathrm{~m}^{3}$

Parámetros de diseño:

- Presión máxima $290 \mathrm{mb}$

- Temperatura interior $170{ }^{\circ} \mathrm{C}$

- Temperatura exterior $43^{\circ} \mathrm{C}$

Enfriamiento con nitrógeno líquido $\mathrm{a}-196^{\circ} \mathrm{C}$ y $\mathrm{GNL}$ $\mathrm{a}-163^{\circ} \mathrm{C}$.

\footnotetext{
*IBERINSA (Ibérica de Estudios e Ingenieria, S. A.)

* Director Departamento de Ingeniería SNCMP (Société Nouvelle Constructions Metalliques de Provence)
}

Boil-off

El boil-off máximo garantizado corresponde a una potencia de $79 \mathrm{kw}$, es decir, 0,05\% de boil-off.

\section{Hipótesis específicas de cálculo:}

Seismo - Caso normal: OBE (Operating Basis Earthquake) $a=0,28 \mathrm{~g}$.

- Caso extraordinario: SSE (Safe Shutdown Earthquake) $a=0,34 \mathrm{~g}$,

$a=$ aceleración equivalente.

\section{Burn-out}

El depósito exterior deberá contener el GNL en caso de incendio interior durante $72 \mathrm{~h}$, hasta su total extin- 
ción, comprobando la profundidad máxima de grieta de forma que no sea pasante.

\section{Cold-spot}

El depósito exterior deberá soportar en condiciones de servicio una fuga predeterminada del depósito interior.

\section{Fuego exterior}

El depósito exterior deberá soportar en condiciones de servicio durante seis horas un fuego externo de $12 \mathrm{kw} / \mathrm{m}^{2}$.

\section{Fisuración}

Se limitará en operación a 0,1 mm en el hormigón de muro y cúpula.

Impacto

El depósito exterior deberá soportar sin penetración el impacto a $50 \mathrm{~m} / \mathrm{s}$ de una masa de $2.000 \mathrm{~kg}$ en muro y de $1.000 \mathrm{~kg}$ en cúpula.

\section{Condicionantes del emplazamiento:}

\section{Climáticos}

La situación del tanque en una zona sometida a altas temperaturas, ha obligado a diseñar un aislamiento importante del depósito interior, tal que minimice la tasa de formación de gas dentro del tanque por ganancia de calor.

\section{Geotécnicos}

El estudio geotécnico ha permitido elegir una cimentación directa mediante losa circular, apoyada sobre un relleno de grava compactada' en sustitución del terreno natural, al estar compuesto el suelo en sus primeros metros por arenas poco densas.

\section{Sísmicos}

El emplazamiento del depósito en zona de influencia sismica de la falla Azores-Gibraltar, y la consideración del tipo de producto almacenado, ha supuesto la necesidad de efectuar un cálculo sísmico muy riguroso mediante elementos finitos, modelizando el conjunto tanque interior - tanque exterior - suelo, para realizar el análisis modal, partiendo de espectros de respuesta de diseño con obtención de los modos de vibración y factores de participación modal, y finalmente mediante integración directa obtener los espectros de respuesta a considerar en distintos puntos del modelo.

\section{DEPÓSITO EXTERIOR}

\subsection{Características del depósito}

El depósito exterior consiste en un depósito cilíndrico de hormigón postesado, completamente cerrado, formado por una losa de cimentación, un muro de hormigón y la cúpula esférica, que rodea completamente al depósito interior.

La función del depósito exterior es doble:

Por una parte sirve como cimentación del depósito interior, y por otra parte constituye el recipiente secundario o de seguridad para el caso de rotura o derrame del depósito interior y frente a acciones exteriores.

Los elementos de que consta el depósito exterior son:

- Losa de cimentación de hormigón armado.

- Muro de hormigón postesado.

- Cúpula de hormigón armado.

En él se encuentran la entrada provisional a través del muro y la estructura de la plataforma para bombas, situada sobre la cúpula.

Las dimensiones principales del depósito, cuya capacidad geométrica es de $82.000 \mathrm{~m}^{3}$, pueden verse en la (Fig. 1).

Las principales características de los materiales utilizados en el depósito exterior son:

- Hormigones: losa de cimentación .............. H-300

muro $\mathrm{H}-400$

cúpula $\mathrm{H}-300$

- Acero para pretensar. Carga unitaria de rotura $190 \mathrm{~kg} / \mathrm{mm}^{2}$.

- Acero pasivo AEH-500 CRIO (denominación dada al tipo de acero nacional desarrollado expresamente para este proyecto y capaz de cumplir las especificaciones del mismo sobre ensayos a $-170^{\circ} \mathrm{C}$ ).

\subsection{Consideraciones de diseño}

\section{a) Cimentación}

De acuerdo con las conclusiones del Informe preliminar, contrastadas por medio de ensayos de penetración dinámica realizados durante el diseño, el terreno de cimentación consiste en arenas densas o muy densas, homogéneas en todo el emplazamiento del tanque, observándose únicamente en algunos de los sondeos per- 
forados que la capa más superficial de uno o dos metros de espesor presenta una densidad menor, floja a medianamente densa.

En dicho informe se comenta igualmente que, con el fin de evitar o compensar la ligera heterogeneidad en la densidad de las arenas de la capa más superficial, se considera suficiente con excavar en el emplazamiento del tanque hasta la cota $+3,50 \mathrm{~m}$, es decir, entre uno o dos metros y volver a colocar el mismo material com. pactándolo adecuadamente.

Aunque la mejor cota de cimentación, desde el punto de vista geotécnico, sería la citada $+3,50$, otros condicionantes derivados de la explotación obligan a que la losa se cimente a una cota más alta.

Teniendo en cuenta esto, se ha proyectado una cimentación sobre un relleno de grava compactada por encima de la cota $+3,50$, a la que se llega previa excavación del terreno natural existente. En la zona central del depósito el relleno compactado de material granular puede ser más arenoso.

La razón de poner un relleno de grava en el anillo perimetral de la losa es evitar las socavaciones en caso de una hipotética fuga del gas líquido.

En el exterior del depósito se proyecta un dren con el fin de evitar que el nivel freático alcance el fondo de la losa de cimentación, con el consiguiente peligro de congelación.

La cimentación consiste en una losa circular de hormigón armado y postesado de 54,01 m de diámetro, en la que se pueden distinguir tres zonas: una central circular de espesor mínimo y constante, otra perimetral exterior en anillo de espesor máximo también constante bajo el muro pretensado del depósito, y otra intermedia con espesor variable.

Dentro de este último anillo se proyecta una junta de construcción, a hormigonar una vez pretensada la zona perimetral y la parte más baja del muro.

Para el anclaje de los tendones de la losa de cimentación se disponen cuatro contrafuertes exteriores, repartidos a $90^{\circ}$, y en la misma vertical que los contrafuertes del muro.

\section{b) Muro}

El espesor del muro es constante e igual a $0,70 \mathrm{~m}$, salvo en la parte inferior de empotramiento en la losa de cimentación donde se ha aumentado a $1,50 \mathrm{~m}$, uniéndose en su parte superior monolíticamente con la cúpula esférica.
El muro está pretensado tanto en dirección horizontal como en la vertical.

El pretensado horizontal lo constituyen anillos completos, distribuidos en toda la altura del muro con separación variable, y formados cada uno por dos tendones de $180^{\circ}$ que se anclan en cuatro contrafuertes situados en los extremos de dos diámetros perpendiculares. Cada anillo de pretensado está girado $90^{\circ}$ respecto a los dos adyacentes.

Los tendones son todos iguales con las siguientes características:

- Tipo: $19 \varnothing 0,5 "$.

- Calidad: según ASTM A-416.

- Carga unitaria de rotura $190 \mathrm{~kg} / \mathrm{mm}^{2}$.

- Carga de rotura $360 \mathrm{t}$.

- Fuerza de anclaje 249,2 t.

- Diámetro de la vaina $90 \mathrm{~mm}$.

- Longitud $84,00 \mathrm{~m}$.

El pretensado vertical se logra con una serie de tendones, repartidos uniformemente. Los tendones verticales tipo tienen un trazado en $\mathrm{J}$, con el anclaje superior en la parte más alta del mura y el inferior en el escalón que forma el tacón existente en la base del mismo. En planta los tendones están girados para salir casi tangentes al muro, y obtener asi una curvatura admisible que permita su enfilado. El punto más bajo de estos tendones queda al nivel de la armadura inferior de la viga anillo de cimentación del muro. Los tendones cuyo anclaje inferior queda en la zona de los contrafuertes del muro tienen su trazado en $\mathrm{L}$, anclándose en los mismos tacones que sirven para el anclaje de los tendones de pretensado de la viga anillo de cimentación (Fig. 2, pág. 16).

Los tendones verticales se tesan desde ambos extremos, con la misma calidad de los del pretensado horizontal y las siguientes características:

- Sección: $15 \varnothing$ 1/2".

- Carga de rotura: $281 \mathrm{t}$.

- Fuerza de anclaje: $197 \mathrm{t}$.

- Longitud: 46,50 y $48,50 \mathrm{~m}$.

- Diámetro de la vaina: $81 \mathrm{~mm}$.

Para el acceso al interior del depósito, durante la construcción, se han previsto dos entradas provisionales en el muro, situadas a la misma altura y diametralmente opuestas. Una de éllas con dimensiones que permiten la entrada de materiales y accesorios y otra reducida, como salida de emergencia. 


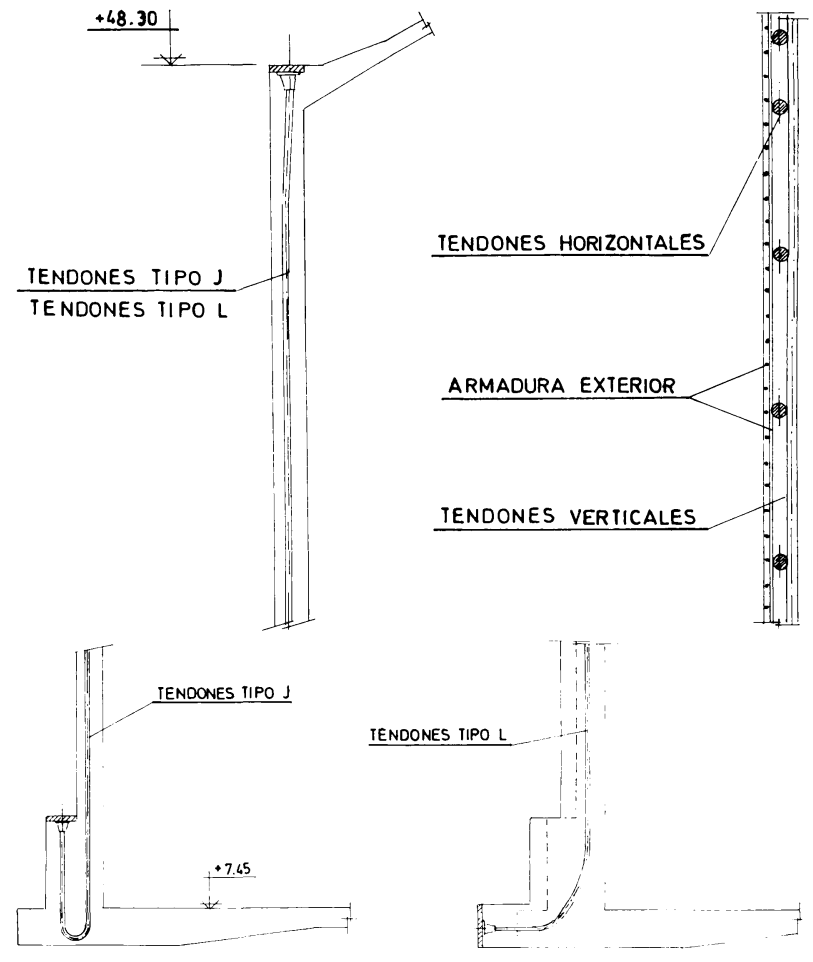

Fig. 2

Estas entradas se mantienen abiertas durante toda la obra, cerrándose después de realizar la prueba hidroneumática del tanque. Para esta prueba se cierran ambas entradas con unas compuertas metálicas, dimensionadas para resistir la sobrepresión interior a que se somete el depósito durante la misma.

Dadas las dimensiones de las puertas provisionales y la separación de los tendones tanto verticales como horizontales, quedan interrumpidos algunos anillos de pretensado horizontal y tendones verticales.

La condición exigida en las especificaciones del depósito es que, a la zona del muro afectada por las puertas, se le aplique un pretensado tal que se consiga un estado de compresiones similar al del resto del muro. Por ello se admitió mantener el trazado de los tendones como si no existieran los huecos de las puertas, interrumpiéndolas al llegar al hueco, para una vez terminadas las fases constructivas en que son necesarias las puertas colocar las vainas, hormigonar los huecos y pretensar los tendones de la franja afectada, es decir, los directamente cortados en los huecos, más aquellos tendones próximos necesarios para obtener el efecto de pretensado necesario.

Toda la superficie interior del depósito exterior está cubierta por una camisa de chapa de acero (liner), para asegurar la estanquidad al gas.

Sobre la solera de hormigón se dispone una chapa de acero sobre la cual se colocan a su vez las capas de bloques de Foam-Glass para aislamiento térmico.
En la pared interior del muro la camisa de chapa es más delgada y se fija al muro soldándola a unas pletinas en forma de malla que se dejan embebidas en el muro al hormigonar.

En la cúpula, la chapa se dimensiona para resistir el peso del hormigón dotándola de conectores para colaborar con el hormigón en la resistencia de la cúpula.

El pretensado del depósito exterior se ha aplicado en distintas fases, según las operaciones del proceso constructivo y pruebas a que se le ha sometido.

Las fases principales de pretensado son las siguientes:

Fase 1.a: Losa de cimentación y tendones horizontales de la zona baja del muro, excepto los situados en la banda de las puertas de acceso.

Fase 2. : Tendones verticales en un $50 \%$.

Fase 3. ${ }^{a}$ : Tendones horizontales en la zona superior del muro.

Fase 4.: Tendones del anillo de compresión de la cúpula.

Fase 5. ${ }^{\mathrm{a}}$ : Tendones horizontales de la zona media del muro.

Fase 6. ${ }^{\mathrm{a}}$ : Tendones verticales restantes, excepto los afectados por las entradas provisionales.

Fase 7. ${ }^{\mathrm{a}}$ : Tendones horizontales restantes de la zona baja del muro, excepto en las puertas.

Fase 8. ${ }^{a}$ : Tesado provisional de los tendones horizontales de las puertas para la prueba hidroneumática del tanque.

Fase 9. : Tesado final de los tendones de las puertas provisionales tanto horizontales como verticales.

\section{c) Cúpula}

El depósito exterior se cierra con una cúpula esférica de $49,41 \mathrm{~m}$ de radio interior, empotrada monolíticamente en la parte superior del muro.

La cúpula es de hormigón armado, de espesor constante, excepto en el anillo de empotramiento en el muro, donde aumenta el espesor gradualmente.

Para el hormigonado de la cúpula, en lugar de cimbrar, se ha montado una cúpula metálica de chapa de acero con rigidizadores y conectores que sirve a la vez como "liner" interior del depósito y como elemento resistente de la estructura de la cúpula.

El empuje horizontal transmitido por la cúpula sobre la parte superior del muro se equilibra por medio de 
un pretensado formado por tendones, de las mismas características que los de pretensado del muro, colocados en el anillo inferior de la cúpula y en la misma vertical del muro, sin sobresalir del mismo para facilitar su construcción con el mismo enconfrado utilizado en el muro.

Con el pretensado aplicado en el anillo de compresión de la cúpula se eliminan los esfuerzos de tracción que de otra forma se producirian en la zona anular perimetral de la cúpula, consiguiéndose que con cargas permanentes toda la cúpula esté en compresión tanto en dirección radial como circunferencial.

Sobre la cúpula se construye una plataforma para las bombas del tanque formada por una estructura metálica sobre un total de 20 pilares.

La cúpula se proyecta con agujeros para las bombas y demás equipos necesarios para el funcionamiento del tanque.

El acceso a la parte superior del tanque se consigue mediante una escalera metálica exterior, adosada al muro.

\subsection{Hipótesis específicas}

El tanque exterior se proyecta de acuerdo con lo exigido en las especificaciones teniendo en cuenta las acciones siguientes:

\section{- Fuego exterior}

El fuego exterior se considera como una radiación de calor de $12 \mathrm{kw} / \mathrm{m}^{2}$ durante 6 horas.

Una estructura de hormigón sometida al fuego, sufre un deterioro superficial que aumenta con el tiempo. Además, el incremento diferencial de temperatura en la estructura produce tensiones anormales y puede reducir las propiedades del hormigón, del acero pasivo y de los cables de pretensado. Se observa que si el fuego durara más de diez horas, la temperatura alcanzada por los aceros reduciría considerablemente la resistencia del tanque de hormigón.

Este efecto no es catastrófico sin embargo para un depósito autoportante de este tipo, ya que el tanque interior conserva toda su integridad.

\section{- Fuego en el interior del tanque}

El análisis del caso de incendio en el interior del tanque se desarrolla en dos etapas. En la primera etapa se determinan las temperaturas en la pared del depósito; estas temperaturas se usan en la segunda etapa

\section{EVOLUCION DE TEMPERATURA EN MURO EXTERIOR}
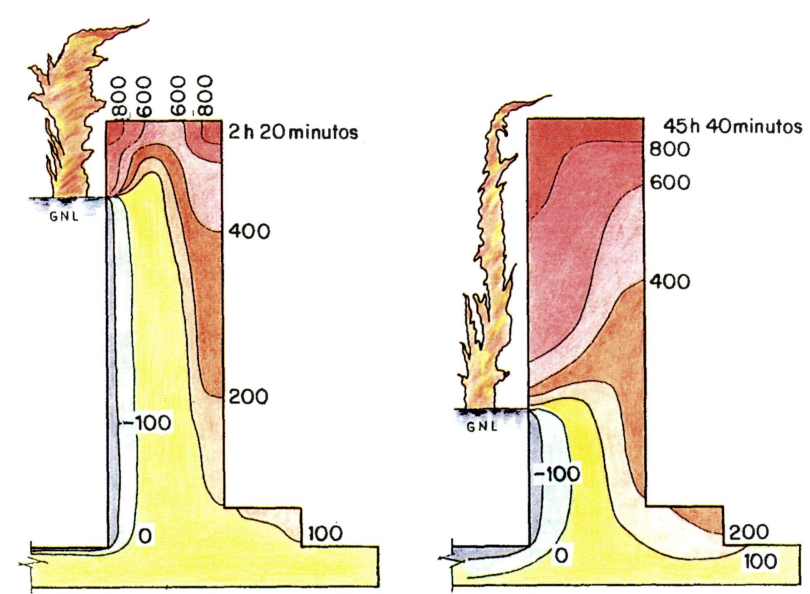

Fig. 3

para obtener las tensiones y fisuración de la pared del depósito.

Para la determinación de temperaturas se utiliza un programa de ordenador que obtiene las temperaturas en los nudos de una estructura bidimensional o axilsimétrica por análisis matricial y desarrollos de Fourier, bajo la acción de un efecto térmico variable en el tiempo.

Los datos de entrada son: el flujo de calor originado en la superficie de GNL, cuyo nivel varía a lo largo del tiempo; la duración del incendio (72 horas según las especificaciones del proyecto); los mecanismos de transmisión del calor, y el comportamiento de los materiales del modelo.

El cálculo da como resultado las temperaturas a través de la pared a distintas horas (Fig. 3).

En la segunda fase del cálculo, con un programa de elementos finitos se obtienen tensiones y fisuración en el hormigón bajo la acción de las cargas y el efecto térmico.

Los datos de entrada son: el peso de la estructura, la presión debida al GNL y las temperaturas obtenidas en la fase anterior.

Para el cálculo se considera el módulo de elasticidad del hormigón y el límite elástico de los aceros variables con la temperatura.

Los resultados del programa dan la solución de la fisuración del muro a distintas horas, desde 2 hasta 72 horas, confirmando la capacidad del muro para contener el GNL durante todo este tiempo. 


\section{- Efectos térmicos de un derrame de GNL}

Se considera en el diseño, junto con la presión hidrostática del GNL derramado, el gradiente térmico producido en el hormigón por el contacto del GNL a temperatura criogénica.

Para este análisis se considera el fuerte gradiente térmico a que está sometida la pared de hormigón.

Este gradiente térmico da lugar a zonas de la estructura donde el material se comporta de forma no elástica originándose fisuras en las zonas traccionadas del hormigón.

Para el estudio del tanque se considera en primer lugar la propagación de la temperatura en el tiempo a través de la estructura. A continuación se estudia el depósito considerando el comportamiento no lineal del hormigón y el acero para obtener las zonas en que se produce la fisuración del mismo, y en consecuencia, la capacidad del depósito para contener el GNL derramado.

\section{DEPÓSITO INTERIOR}

\subsection{Características}

es el resultante de los aspectos de respuesta de los terremotos OBE y SSE definidos para este proyecto.

Para el análisis sísmico se parte de las propiedades del terreno, según los datos aportados por el Informe Geotécnico. Mediante el programa de ordenador "SHAKE" de la Universidad de California en Berkeley se obtienen los datos de deformación del terreno, necesarios para definir las funciones de impedancia del mismo.

Una vez definido el terreno, con el programa SAP-IV se analiza la estructura discretizándola en elementos tipo lámina, tipo sólido y elementos de cotorno para obtener los modos de vibración dinámica (Fig. 4) y espectros de respuesta en distintos niveles de la estructura.

Los componentes horizontales y verticales del mismo se combinan con doble signo para la componente vertical.

El conjunto suelo-estructura se estudia de tal manera que los elementos más solicitados pueden resistir las tensiones asi determinadas.

Además, la respuesta de la estructura a diferentes niveles, permitirá estudiar correctamente los accesorios del tanque, en particular el conjunto de tuberías y sus soportes.
Diámetro (en caliente) $47,21 \mathrm{~m}$.

Altura $37,31 \mathrm{~m}$.

Espesor mínino $8,7 \mathrm{~mm}$.

Espesor máximo $24,9 \mathrm{~mm}$.

\subsection{Distinción de los elementos del tanque}

El código API $620 \mathrm{Ap} Q$ distingue dos tipos de componentes para el eśtudio del tanque:

- Los compønentes primarios, que son aquellos que están:

- en contacto con el líquido almacenado;

- sometidos a tensiones;

- sometidos a baja temperatura;

- sometidos a choques térmicos.

- Los componentes secundarios, que son los que:

- no tienen grandes tensiones;

- no provocan fuga de líquido, en caso de rotura;

- están en contacto con el vapor y, por tanto, la temperatura de diseño es superior a $-60^{\circ} \mathrm{F}$. 
Las paredes y el fondo están por tanto consideradas como componentes primarios.

El techo suspendido puede clasificarse como componente secundario.

\subsection{Tipos de aceros y tensiones de diseño}

- Se ha empleado el tipo de acero ASTM A553 (QT).

- Las tensiones admisibles quedan definidas por las de las uniones soldadas. Se tomará el menor de los dos valores siguientes:

$1 / 3 \mathrm{~T} . \mathrm{S}=1 / 3.662 \mathrm{~N} / \mathrm{mm}^{2}=220,7 \mathrm{~N} / \mathrm{mm}^{2}$

$2 / 3 \mathrm{Y} . \mathrm{S}=2 / 3.400 \mathrm{~N} / \mathrm{mm}^{2}=266,7 \mathrm{~N} / \mathrm{mm}$

T.S = Tensión de rotura

Y.S = Limite elástico

Para la prueba hidráulica, se verificará que las tensiones son inferiores a:

$0,85 \mathrm{Y} . \mathrm{S}=0,85.400 \mathrm{~N} / \mathrm{mm}^{2}=\underline{340 \mathrm{~N} / \mathrm{mm}^{2}}$

$0,55 \mathrm{~T} . \mathrm{S}=0,55.662 \mathrm{~N} / \mathrm{mm}^{2}=364,1 \mathrm{~N} / \mathrm{mm}^{2}$

Los valores subrayados son por tanto los tenidos en cuenta para el diseño, siendo uno u otro, según el tipo especificado de prueba hidráulica.

La prueba hidráulica puede ser parcial o total según que el volumen de agua sea inferior o igual a la capacidad nominal del depósito.

Para prueba parcial, la cantidad de agua considerada es generalmente 1,25 veces el peso del producto almacenado.

Por tanto, normalmente no es éste el tipo de prueba dimensionante para el espesor de las chapas.

Para que lo sea, la prueba debe hacerse con una cantidad de agua superior a 340/220,7 $=1,54$ veces el peso del producto.

Por encima de este valor es la prueba hidráulica la que condiciona el diseño de las virolas del depósito. Es el caso del tanque de Huelva, ya que la prueba hidráulica ha sido total.

Además de las virolas, otros elementos importantes de diseño han sido el rigidizador de cabeza del tanque, los rigidizadores de las virolas para contener la presión exterior de la perlita y los anclajes para impedir el levantamiento del depósito en las hipótesis de seismo.

\section{AISLAMIENTO}

El criterio básico para diseñar el aislamiento de un depósito criogénico es la tasa de evaporación diaria (boiloff), es decir, la cantidad de líquido evaporado al día (24 horas), expresando en porcentaje de la capacidad total del depósito.

El valor generalmente aceptado está comprendido entre $0,05 \%$ y $0,1 \%$. En el caso del depósito de Huelva, $0,05 \%$.

Para obtener este "boil-off" es necesario, por tanto, aislar correctamente el tanque interior.

El aislamiento del fondo debe ser al mismo tiempo capaz de resistir las presiones del tanque interior, por lo que debe tener una buena resistencia a la compresión.

Se ha resuelto mediante $700 \mathrm{~mm}$ de vidrio celular (Foam-Glass).

El tanque interior se apoya en un anillo de hormigón especial, ligero y de baja conductividad, con bolas de vidrio expandido como árido.

Sobre el techo suspendido, el aislamiento se realiza por medio de $700 \mathrm{~mm}$ de lana de vidrio.

Para aislar las virolas se coloca una manta de lana de vidrio elástica sobre la cara exterior del tanque interior. El espacio anular libre, comprendido entre la manta resiliente y el tanque exterior de hormigón, se llena con perlita expandida (un material ligero y de escasa conductividad) vibrada posteriormente.

\section{EQUIPAMIENTO}

\subsection{Instalaciones e Instrumentación}

Finalmente, para el correcto funcionamiento del tanque se ha diseñado el equipamiento, cuyos elementos principales se relacionan a continuación:

\subsection{Tuberías}

Las líneas principales son las entradas y salidas del producto (líquido o vapor):

- Entradas:

Alta y baja con accesorios de mezcla. Esto permite al usuario, según las calidades del producto almacenado en el tanque y por llegar, favorecer la mezcla y evitar el fenómeno de "Roll Over" que podría eventualmente ocurrir. 
- Salidas:

Para el GNL se han utilizado 4 bombas sumergidas con una más de reserva situada en el fondo del tanque, dentro de las tuberías de vaciado (pozos de bombas).

Una salida de vapor para el "boil-off".

Varias salidas de vapor de seguridad, hacia las respectivas válvulas de seguridad, de presión o de vacío.

Otras líneas son las de puesta en servicio del tanque, puesta en frio, inertizado y purga o calentamiento del tanque.

\subsection{Equipos de control y seguridad}

Merecen destacarse los siguientes:

- Medidores de nivel con alarmas de nivel, alto y bajo, que permiten la parada de la alimentación del tanque, o la parada de las bombas en caso de nivel bajo.

- Válvulas de seguridad de presión y vacío.
Sus condiciones de diseño son:

- Presión:

- Boil-off.

- Disminución de la presión atmosférica.

- Llegada del producto caliente con "flash".

- Fuego externo.

- Vacio:

- Vaciado demasiado rápido.

- Aumento de presión atmosférica.

- Compresor de "boil-off" demasiado potente.

- Sistema contraincendios.

- Medidores de temperatura para el tanque y para el producto.

- Medidores de presión.

- Medidores de densidad de temperatura a diferentes niveles y registradores de datos. Estos medidores permiten tomar las medidas necesarias para la homogeneidad del producto $y$ reaccionar antes de que se produzca un "roll-over".

\title{
GEHO
}

EI GRUPO ESPAÑOL DEL HORMIGÓN (GEHO) se complace en invitarle al Seminario referente al EUROCÓDIGO-2: ESTRUCTURAS DE HORMIGÓN, que se celebrará el día 7 de junio de 1989, a partir de las 09,30 horas, en la Sala de Conferencias del Instituto de la Construcción y del Cemento "Eduardo Torroja", del Consejo Superior de Investigaciones Científicas.

\author{
Información: \\ Sra. Rosa Rodríguez \\ Cl. Serrano Galvache, s/n. (acceso por Arturo Soria, fren- \\ te al número 278). \\ Teléfono (91) 2020440 - 28033 MADRID
}

